Mycologia, 96(3), 2004, pp. 667-674.

(C) 2004 by The Mycological Society of America, Lawrence, KS 66044-8897

\title{
Acanthostigma and Tubeufia species, including T. claspisphaeria sp. nov., from submerged wood in Hong Kong
}

\author{
Rampai Kodsueb \\ Saisamorn Lumyong ${ }^{1}$ \\ Department of Biology, Faculty of Science, Chiang Mai \\ University, Chiang Mai, Thailand \\ Pipob Lumyong \\ Department of Plant Pathology, Faculty of Agriculture, \\ Chiang Mai University, Chiang Mai, Thailand \\ Eric H.C. McKenzie \\ Landcare Research, Private Bag 92170, Aukland, \\ New Zealand \\ Wai Hong Ho \\ Kevin D. Hyde \\ Centre for Research in Fungal Diversity, Department of \\ Ecology Ẽ Biodiversity, The University of Hong Kong, \\ Pokfulam Road, Hong Kong
}

\begin{abstract}
Acanthostigma scopulum, Tubeufia claspisphaeria sp. nov. and T. paludosa were identified from submerged wood collected in a small forest stream on Lantau Island, Hong Kong. The collections of Acanthostigma scopulum and Tubeufia paludosa differed slightly from the original descriptions. Tubeufia claspisphaeria differs from previously described species in that it has hook-shaped setae that form radially around the ostiole. This new species is described and illustrated and compared with the most similar species. A dichotomous key to the 16 accepted species in Tubeufia is provided.

Key words: new species, saprobic fungi, systematics
\end{abstract}

\section{INTRODUCTION}

There are 22 genera in the Tubeufiaceae (Barr 1980, Rossman 1987, Kirk et al 2001). Some genera, such as Melioliphila and Uredinophila, are hyperparasites on sooty molds and on rust fungi, while Podonectria is parasitic fungi on scale insects. Acanthostigma and Tubeufia are saprobes, usually found on old, rotten wood. Previous examinations of freshwater fungi occurring on submerged wood in streams in the tropics and subtropics have yielded numerous novel fungi,

\footnotetext{
Accepted for publication October 20, 2003.

${ }^{1}$ Corresponding author. E-mail: scboi009@chiangmai.ac.th
}

including hyphomycetes (Hyde et al 2002, McKenzie et al 2002), coelomycetes (Hyde 1993) and ascomycetes (Wong and Hyde 1999, Cai et al 2002). During our survey of fungi occurring on naturally submerged wood, we collected an Acanthostigma and two Tubeufia species, one being a species new to science, from a small forest stream in Hong Kong. The three species are described, illustrated and compared with known taxa. A key to the 16 accepted species of $T u$ beufia is provided.

\section{MATERIALS AND METHODS}

Submerged wood was retrieved from a stream near the Trappist Monastery, Lantau Island, Hong Kong, in Apr 2002 and returned to the laboratory, where it was incubated in zip-lock plastic bags at room temperature. Humidity was maintained by adding moistened paper towels. Samples were examined within $3 \mathrm{~d}$ and periodically over 1 mo for the presence of sporulating structures. Cultures of fungi were obtained where possible from single spores (Choi et al 1999). To stimulate sporulation, $1 \mathrm{~cm}^{2}$ blocks of colonized agar were placed in water in a bubble chamber overnight.

\section{TAXONOMY}

Acanthostigma De Not. and Tubeufia Penz. \& Sacc. Acanthostigma currently includes six species and recently has been reviewed by Réblová and Barr (2000). The genus is characterized by vinaceous, reddishbrown or dark brown ascomata that are covered with dark brownish-black, often opaque, obtuse or acute setae. The ascospores are hyaline, multiseptate and cylindrical-fusiform to elongate fusiform. The anamorphs are in Helicosporium and Helicomyces. In species of Tubeufia the ascomata are hyaline, whitish or yellowish to pinkish, but may become dark at maturity. They are smooth, or are covered with protruding cells, thick-walled hyphal appendages, or short dark setae (Réblová and Barr 2000).

Acanthostigma scopulum (Cooke \& Peck) Peck, Bull.

New York State Mus. 1:22 (1887) (Figs. 1-6)

Ascomata 145-250 $\mu \mathrm{m}$ diam, superficial, globose, solitary or gregarious, dark brown to black, membranous, ostiolate, with setae; setae (56-)80-90(-99) $\mu \mathrm{m}$ long, 4-6 $\mu \mathrm{m}$ wide at base, 1.5-2 $\mu \mathrm{m}$ wide at apex, dark brown, 1-2-septate, thick-walled, straight. Asci (64-)90-120(-130) × 8-10 $\mu \mathrm{m}$ 

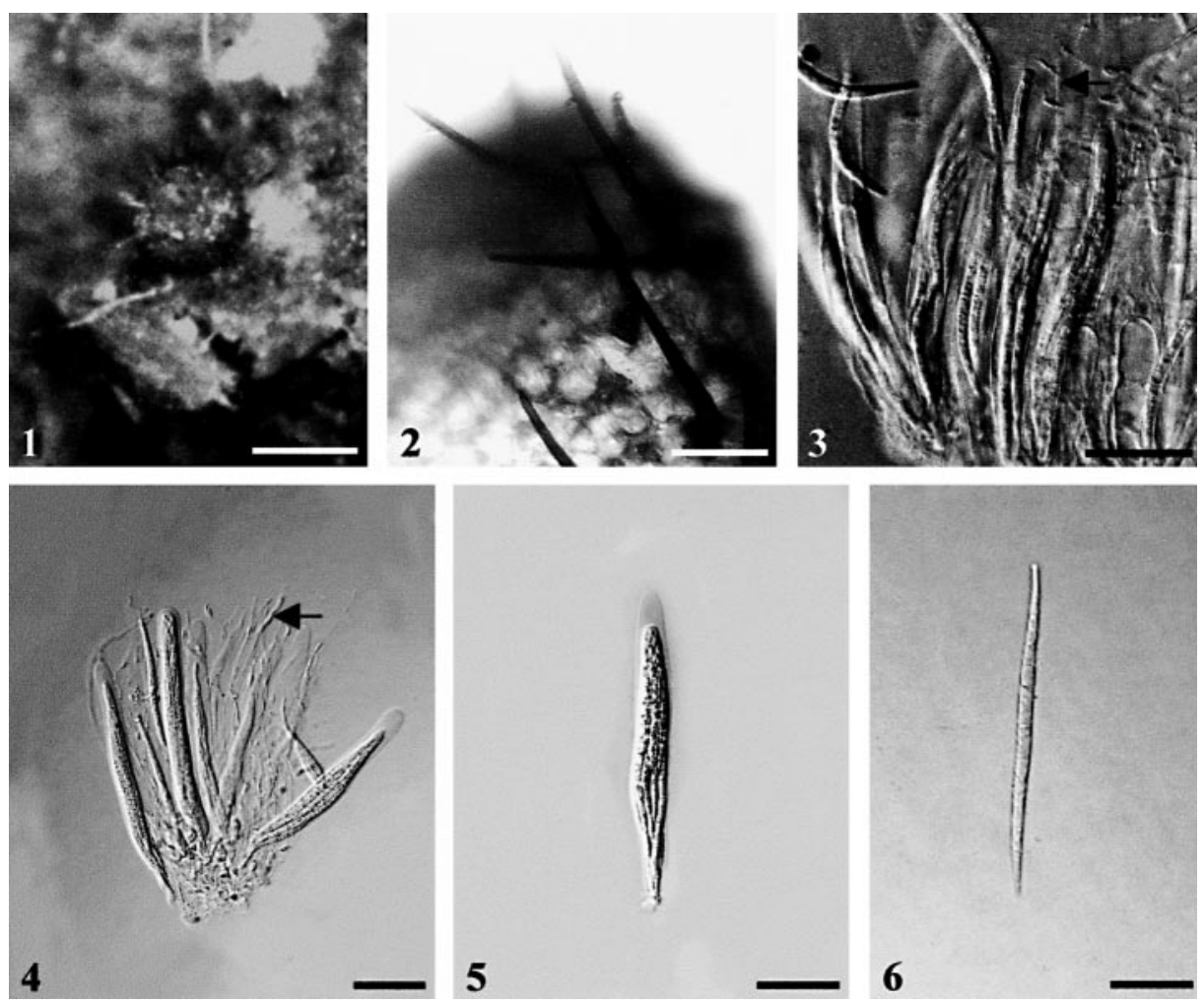

Figs. 1-6. Acanthostigma scopulum (from HKU[M] 17121). 1. Ascomata on natural substratum. 2. Dark brown, septate setae with acute apex. 3-4. A cluster of mature and immature asci. 5. Ascus. 6. Ascospore. Pseudoparaphyses in Fig. 3-4 (arrowed). Bars: $1=200 \mu \mathrm{m} ; 2-5=30 \mu \mathrm{m} ; 6=25 \mu \mathrm{m}$. Figs. 2-3 mounted in water, other figures mounted in lactophenol.

$(\bar{x}=103 \times 9.2 \mu \mathrm{m}, \mathrm{n}=20), 8$-spored, cylindric-clavate, bitunicate, short stalked, pseudoparaphyses. Ascospores (66-) 70-85(-90) $\times 3-3.5(-4) \mu \mathrm{m}(\bar{x}=78 \times 3.4 \mu \mathrm{m}, \mathrm{n}=$ $30)$, fasciculate, long-fusiform to cylindrical-fusiform, hyaline, straight or slightly curved, (5-)9-12-septate, smoothwalled, guttulate, lacking appendages and sheaths.

Anamorph. Helicosporium aureum (Corda) Linder, Ann. Missouri Bot. Gard. 16:279, 1929, from Réblová and Barr (2000).

Colonies on potato-dextrose agar olive-colored, reaching $1 \mathrm{~cm}$ diam in $10 \mathrm{~d}$ at room temperature ( $28 \mathrm{C}$ ), no pigment diffusing into agar, not sporulating even after submergence overnight in a bubble chamber.

Substratum. Wood submerged in streams, decaying wood (Fagus sylvatica, Pinus sp. and Tsuga sp.)

Known distribution. Australia, Europe, Hong Kong and U.S.A.

Specimen examined. HONG KONG. Lantau Island, Trappist Monastery, small stream in forest, on wood partially submerged, 21 Apr 2002, K.D. Hyde (HKU[M] 17121; living culture HKUCC 9117).

Notes. A key to species of Acanthostigma was provided by Réblová and Barr (2000). This collection differs from the description for A. scopulum provided by Réblová and Barr (2000) in that it has septate setae and wider ascospores 3-3.5(-4) $\mu \mathrm{m}$ versus (2-) 2.5-3(-3.5) $\mu \mathrm{m}$ but in other aspects it is similar. Tubeufia claspisphaeria Kodsueb, sp. nov.

(FIGS. 7-16)

Ascomata 185-330(-350) $\mu \mathrm{m}$ diametro, superficialia, globosa, solitaria vel aggregata, hyalina vel pallide brunnea ubi immatura, atrobunnea vel atris ubi maturus, membranea, ostiolata, setosa; setae usque $47 \mu \mathrm{m}$ longa, $10 \mu \mathrm{m}$ lata, (0-) 1(-2)-septata, atrobrunnea, hamatus. Peries ascomati usque $60 \mu \mathrm{m}$ lata crassus, vinacea-brunnea, e cellulis pseudoparenchymatis compositum, 4-5 stratosus, textura globulosa. Asci (93-) 105-120(-127) × (12-) 14-16(-18) $\mu \mathrm{m}$, octospori, cylindrico-clavati, bitunicati, pedicellati. Ascosporae (34-) 45-60(-64) $\times 5-7(-8) \mu \mathrm{m}$, fasciculatae, elongatae-fusiformis, (3-)5-6(-8)-septatae, hyalinae, curvatae, guttulatae.

Etymology. In reference to the clasp-like setae on the ascomata. Ascomata 185-330(-350) $\mu \mathrm{m}$ diam, superficial, globose, solitary or grouped, hyaline to pale brown when immature, dark brown becoming black when mature, membranous, ostiolate, with setae that form around ostiole. Setae from surface of ascomata, up to $47 \mu \mathrm{m}$ long and $10 \mu \mathrm{m}$ wide, (0-) $1(-2)$-septate, dark brown, thick-walled, hook-shaped. Peridium up to $60 \mu \mathrm{m}$ wide, vinaceous brown, comprising 4-5 lay- 

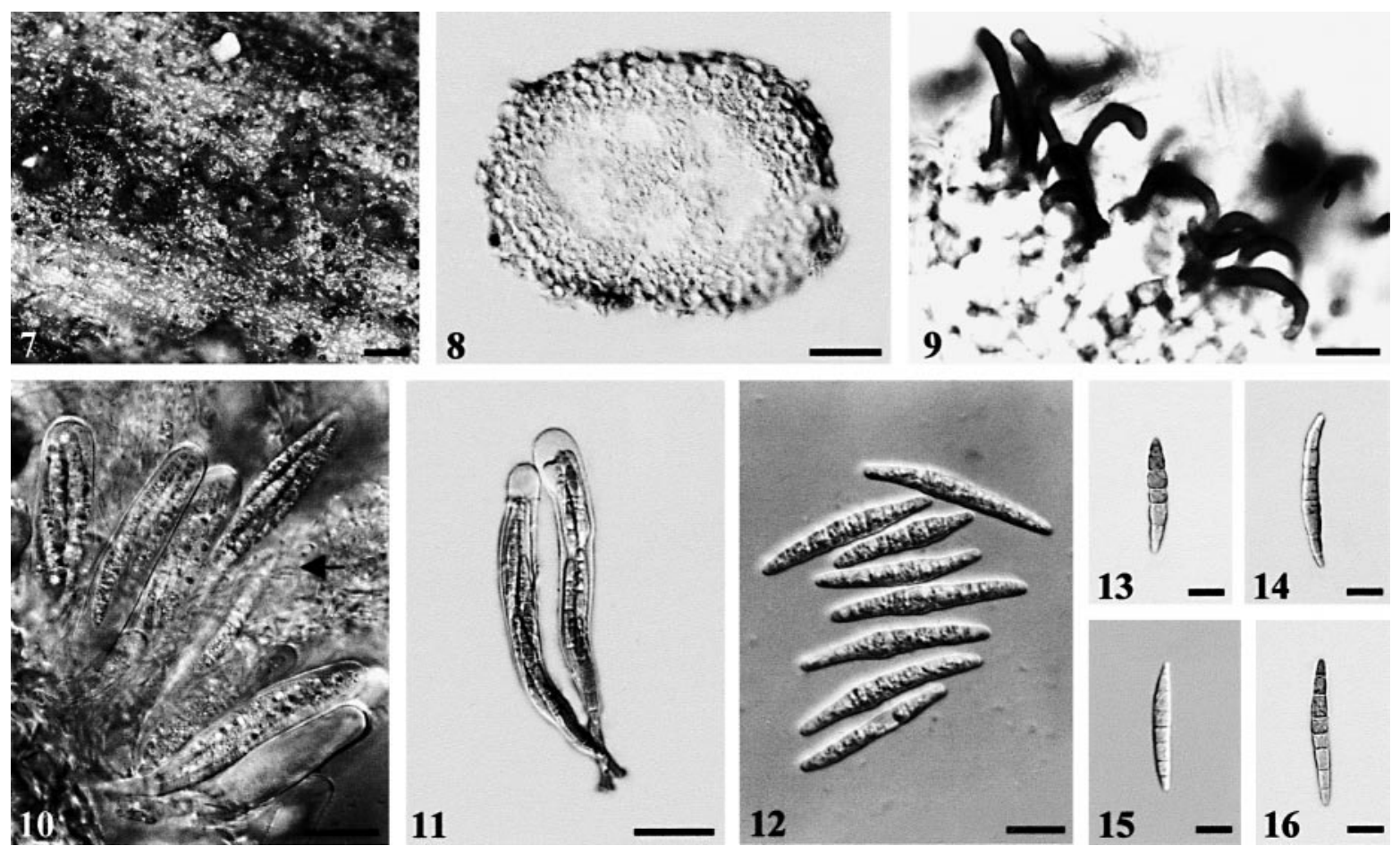

Figs. 7-16. Tubeufia claspisphaeria (from holotype). 7. Ascomata on natural substratum. 8. Longitudinal section through an ascoma. 9. Setae. 10. A cluster of mature and immature asci. 11. Asci. 12-16. Ascospores. Pseudoparaphyses in Fig. 10 (arrowed). Bars: $7=250 \mu \mathrm{m} ; 8=50 \mu \mathrm{m} ; 9=25 \mu \mathrm{m} ; 10-11=30 \mu \mathrm{m} ; 12=15 \mu \mathrm{m} ; 13-16=10 \mu \mathrm{m}$. Figs. $8-10$ and 12 mounted in water, other figures mounted in lactophenol.
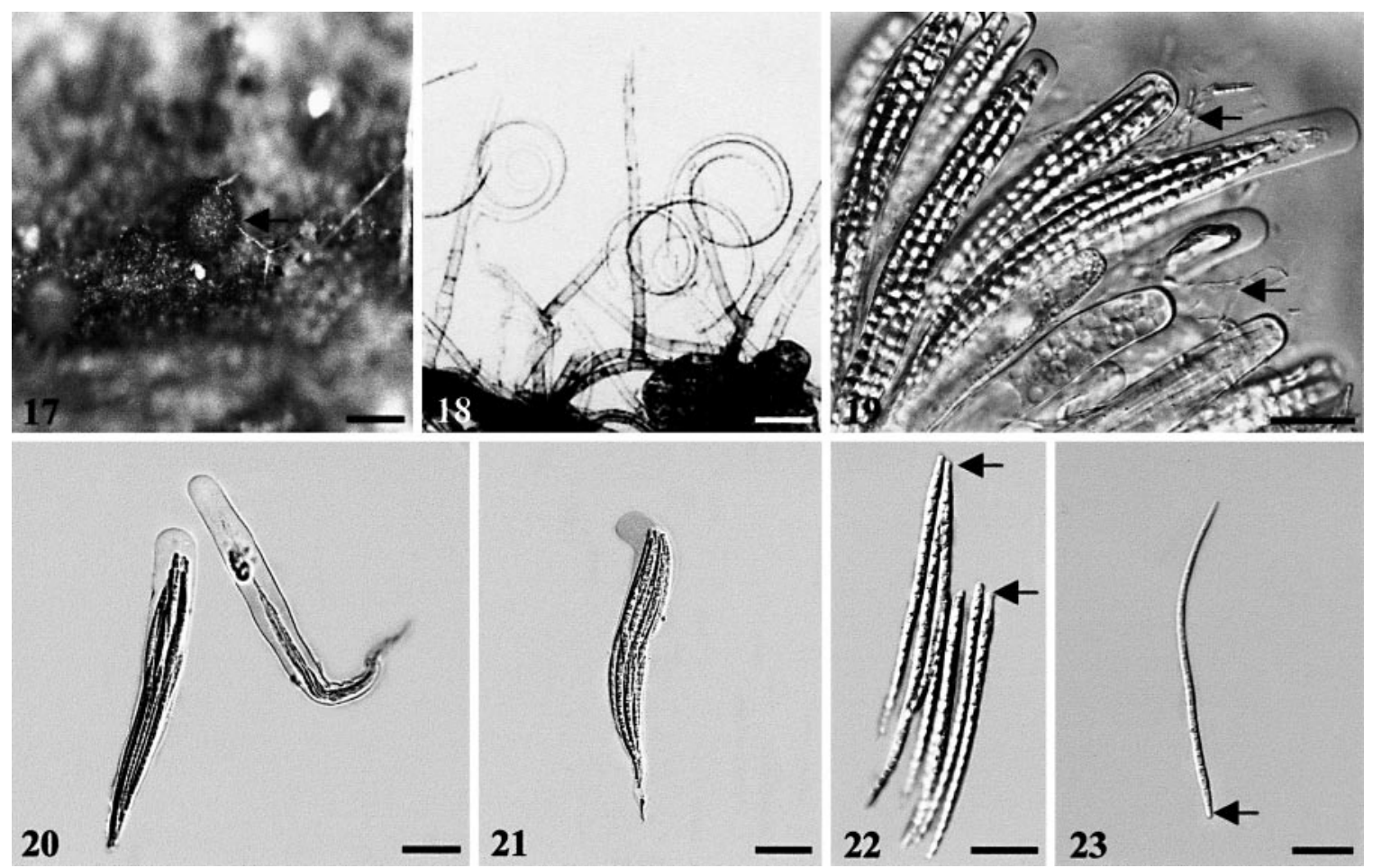

Figs. 17-23. Tubeufia paludosa (from HKU[M] 17122). 17. Ascomata on natural substratum (arrowed). 18. Anamorph (Helicosporium sp.). 19. A cluster of mature and immature asci. 20-21. Asci. 22-23. Ascospores. Pseudoparaphyses in Fig. 19 (arrowed), Mucilaginous pads in Fig. 22-23 (arrowed). Bars: $17=200 \mu \mathrm{m}$; 18-21 = $30 \mu \mathrm{m}$; 22-23 = $25 \mu \mathrm{m}$. Figs. 18, 2021 and 23 mounted in lactophenol, other figures mounted in water. 
TABLE I. Tubeufia species and their present synonyms (accepted name in bold)

\begin{tabular}{|c|c|c|}
\hline Taxa & Synonym & Notes \\
\hline T. acaciae Tilak \& S. B. Kale & None & $\begin{array}{l}\text { Although not examined this taxon may } \\
\text { not be a Tubeufia with its violet to red } \\
\text { ascomata and multiseptate ascospores }\end{array}$ \\
\hline $\begin{array}{l}\text { T. aciculospora Katum. \& Y. Hara- } \\
\text { da }\end{array}$ & None & $\begin{array}{l}\text { This species is unusual as it has asco- } \\
\text { spores with pointed ends Katumoto } \\
\text { and Harada (1979) }\end{array}$ \\
\hline T. albo-ostiolata Rossman & Thaxteriella alboostiolata & Transferred in Crane et al (1998) \\
\hline T. alpina L. Holm \& Nograsek & Acanthostigmina longisporum & $\begin{array}{l}\text { This was considered to be synonymous } \\
\text { with A. longisporum by Réblová and } \\
\text { Barr (2000) }\end{array}$ \\
\hline $\begin{array}{l}\text { T. amazonensis Samuels, Rossman } \\
\text { \& E. Müll. }\end{array}$ & Thaxteriella amazonensis & Transferred in Crane et al (1998) \\
\hline T. anceps Penz. \& Sacc. & Tubeufia paludosa & Synonymized by Rossman (1977) \\
\hline T. asclepiadis Bat. \& Garnier & Saccardomyces socius Henn. & Rossman (1979) \\
\hline $\begin{array}{l}\text { T. aurantiella (Penz. \& Sacc.) Ross- } \\
\text { man }\end{array}$ & $\begin{array}{l}\text { Section Nectrioidea }(=\text { Calonec- } \\
\quad \text { tria aurantiella })\end{array}$ & $\begin{array}{l}\text { Mentioned in Crane et al (1998), trans- } \\
\text { ferred from Calonectria (Rossman } \\
\text { 1979) }\end{array}$ \\
\hline T. brevispina (M. E. Barr \& Roger- & $(=$ Acanthostigmella brevispina $)$ & Crane et al (1998) \\
\hline
\end{tabular}
son) J. L. Crane, Shearer \& M. E. Barr)

T. cerea (Berk. M. A. Curtis) Höhn.

T. clintonii (Peck) M. E. Barr

Section Necrioidea

Acanthostigma perpusillum

T. coccicola (Ellis \& Everh.)

T. coronata Penz. \& Sacc.

T. corynespora Munk

T. cylindrothecia (Seaver) Höhn.

T. dactylariae Chang

T. eriodermae Etayo

T. genuflexa Höhn.

T. hebridensis Dennis

T. helicoma (W. Phillips \& Plowr.) Piroz.

T. helicomyces Höhn.

T. indica (Dharne \& E. Müll.)

Deoray \& Ujjainkar

T. javanica Penz. \& Sacc.

T. minuta Munk

T. miscanthi W. H. Hsieh, C. Y.

Chen \& Sivan.

T. nigrotuberculata T. Hino \& Katum.

T. ovatum Rossman

T. pachythrix (Rehm) Rossman

T. palmarum (Torrend) Samuels, Rossman \& E. Müll.

T. paludosa (P. Crouan \& H. Crouan) Rossman

T. pannariae Etayo

T. parvula Dennis

T. pezizula (Berk. \& M. A. Curtis)

M. E. Barr

T. roraimensis (Samuels \& E. Müll.)

Podonectria coccicola Petch

Tubeufia paludosa

Thaxteriella corynespora

Tubeufia paludosa

None

None

Acanthostigmella genuflexa

Taphrophila hebridensis

Thaxteriella helicoma

Tubeufia paludosa

Thaxteriella indica

Tubeufia paludosa

Herpotrichiella

Taphrophila miscanthi

Thaxteriela ovata

None

Section Nectrioidea

Section Tubeufia

None

None

Thaxteriella pezizula

Thaxteriella roraimensis
Although not examined this taxon may not be a Tubeufia with its violet to red his species is unusual as it has ascospores with pointed ends Katumoto and Harada (1979)

ransferred in Crane et al (1998) Barr (2000)

Transferred in Crane et al (1998)

Synonymized by Rossman (1977)

Rossman (1979)

entioned in Crane et al (1998), trans1979)

Crane et al (1998)

Mentioned in Crane et al (1998), for description see Sivanesan (1984)

This was considered to be synonymous with $A$. perpusillum by Réblová and Barr (2000)

Herpotrichia nigrotuberculata

Rossman (1987)

Synonymized by Rossman (1977)

Transferred in Crane et al (1998)

Synonymized by Rossman (1977)

Chang (2003)

Etayo (2002)-possibly Chaetosphaerulina Barr (1977)

Réblová and Barr (2000)

Transferred in Crane et al (1998)

Synonymized by Rossman (1977)

Mentioned in Crane et al (1998)

Synonymized by Rossman (1977)

Barr (1980)

Réblová and Barr (2000)

Pirozynski (1972)
Transferred in Crane et al (1998)

For discussion see Rossman (1979)

Samuels et al (1979); mentioned in Crane et al (1998)

Samuels et al (1979); mentioned in Crane et al (1998)

Etayo (2002)—possibly Chaetosphaerulina Drawn in Ellis and Ellis (1985)

Type species of Thaxteriela (Sivanesan 1984, Barr 1980)

Samuels and Müller (1978); also mentioned in Crane et al (1998) 
TABLE I. Continued

\begin{tabular}{|c|c|c|}
\hline Taxa & Synonym & Notes \\
\hline T. rugosa C. Booth & Tubeufia helicoma & Barr $(1980)$ \\
\hline $\begin{array}{l}\text { T. scopula (Cooke \& Peck), M. E. } \\
\text { Barr }\end{array}$ & Acanthostigma scopulum & Barr (1980), Réblová and Barr (2000) \\
\hline T. setosa Sivan. \& W. H. Hsieh & Acanthostigma minutum & Réblová and Barr (2000) \\
\hline T. stromaticola (Henn.) Rossman & Transferred from Calonectria & Rossman (1979) \\
\hline $\begin{array}{l}\text { T. trichella (Sacc., E. Bommer \& } \\
\text { M. Rousseau) Scheuer }\end{array}$ & Taphrophila trichela & Réblová and Barr (2000) \\
\hline $\begin{array}{l}\text { T. trichospora (Berk. \& Broome) } \\
\text { Petch }\end{array}$ & Ophionectria trichospora & Rossman (1977) \\
\hline $\begin{array}{l}\text { T. vermicularispora (T. Hino \& Ka- } \\
\text { tum.) Sivan. }\end{array}$ & $\begin{array}{l}\text { Chaetosphaerulina vermicularis- } \\
\text { pora }\end{array}$ & $\begin{array}{l}\text { This has black carbonaceous ascomata } \\
\text { and is probably not a Tubeufia. It was } \\
\text { described as a Chaetosphaerulina (Hino } \\
\text { and Katumoto 1954) }\end{array}$ \\
\hline T. yasudae (T. Hino) Sivan. & Chaetosphaerulina yasudae & Mentioned in Crane et al (1998) \\
\hline
\end{tabular}

ers of thick-walled textura globulosa, outer cells brown-walled, inner cells with hyaline walls. Pseudoparaphyses 1.5-2.5 $\mu \mathrm{m}$ wide, hypha-like, numerous, cellular, unbranched. Asci (93-)105-120(-127) $\times$ (12-) 14-16(-18) $\mu \mathrm{m}(\bar{x}=109 \times 15.1 \mu \mathrm{m}, \mathrm{n}=15)$, 8-spored, cylindric-clavate, bitunicate, with a small ocular chamber, persistently pedicellate. Ascospores (34-) 45-60(-64) × 5-7(-8) $\mu \mathrm{m}(\bar{x}=49 \times 5.9 \mu \mathrm{m}$, $\mathrm{n}=35)$, fasciculate, elongate-fusiform, (3-) 5-6(-8)septate, hyaline, sometimes slightly curved, smoothwalled, guttulate, lacking appendages or sheaths.

Anamorph. Unknown.

Colonies on potato-dextrose agar dark green to black, $1 \mathrm{~cm}$ diam in $1 \mathrm{wk}$ at room temperature ( 28 C). Mycelium mostly immersed, aerial mycelium velvety to fluffy, no pigment diffusing into agar, not sporulating even after submergence overnight in a bubble chamber. Mycelium less dense at the outer edge than in center, with branching mycelial strands extending from edge of colony.

Substratum. Wood submerged in streams.

Known distribution. Hong Kong.

Holotype. HONG KONG. Lantau Island, Trappist Monastery, small stream in forest, on wood partially submerged, 21 Apr 2002, K.D. Hyde (HKU[M] 17123). Living cultures ex holotype HKUCC 9116, HKUCG 9119.

Tubeufia paludosa (P. Crouan \& H. Crouan) Rossman, Mycologia 69:383 (1977)

(FIGS. 17-23)

Ascomata 185-350 $\mathrm{\mu m}$ diam, superficial, globose, brown to dark-brown, solitary, sparse, surface roughened, membranous. Asci (162-)170-180 × (14-)15-
$18(-19) \mu \mathrm{m}(\bar{x}=170 \times 16.1 \mu \mathrm{m} ; \mathrm{n}=17)$, numerous, 8-spored, cylindric-oblong, bitunicate, hyaline, with a subapical ring, rounded at apex, persistently pedicellate, pseudoparaphyses. Ascospores (77-)120$135(-155) \times 4-5(-6) \mu \mathrm{m}(\bar{x}=127 \times 4.3 \mu \mathrm{m}, \mathrm{n}=30)$, fasciculate, narrowly elongate, cylindric or filiform, hyaline, (18-)20-25-septate, straight or slightly curved, smooth-walled, guttulate, with small mucilaginous pads at each end.

Anamorph. Helicosporium sp.

Colonies on potato-dextrose agar $3.5 \mathrm{~cm}$ diam in 3 wk. Mycelium mostly immersed, no pigment diffusing into agar, hyphae less dense in outer zone, sporulating, forming a brownish-gray turf of upright setiform conidiophores, becoming ochraceous or brownish with age; conidiogenous cells produced laterally as thin-walled pegs. Conidia coiled two or three times, multiseptate, coils 37-86.4 $\mu \mathrm{m}$ diam, cells 4.6$5.4 \mu \mathrm{m}$ wide.

Substratum. Wood submerged in streams, decaying woody fruit, palm fruit peduncle and bamboo.

Known distribution. Bermuda, Brazil, Columbia, Europe, Hong Kong, India, Indonesia, Panama, Trinidad, U.S.A. and Venezuela.

Specimen examined. HONG KONG. Lantau Island, Trappist Monastery, small stream in forest, on wood partially submerged, 21 Apr 2002, K.D. Hyde (HKU[M]17122; living culture HKUCC 9118).

The genus Tubeufia has included at least 40 epithets as listed in IndexFungorum (http://www. indexfungorum.org/Names/Names.asp). Many of these taxa have been transferred to other genera (Barr 1980, Crane et al 1998) as summarized in TABLE I. Our two species are typical of Tubeufia because the ascomata are initially pale and have either 


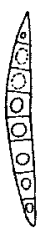

A

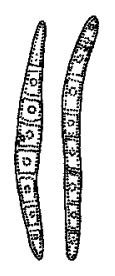

$\mathbf{E}$

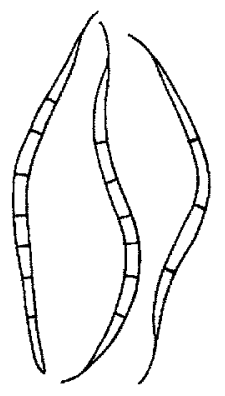

B

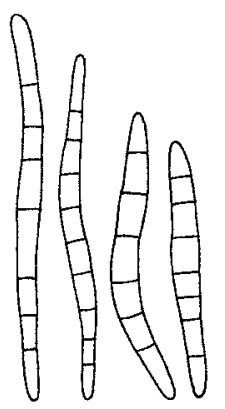

C

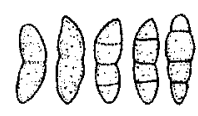

D

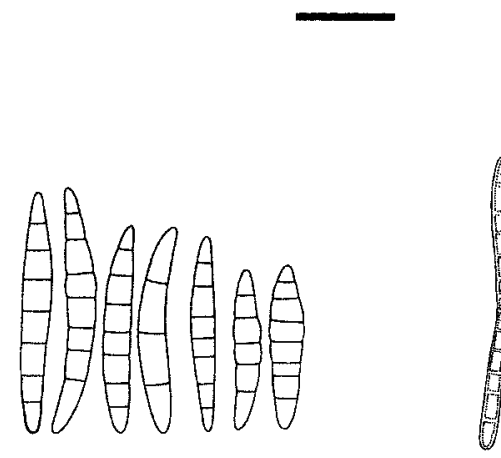

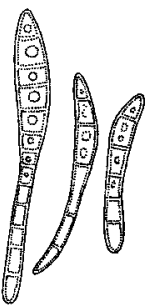

G

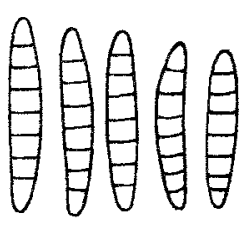

H
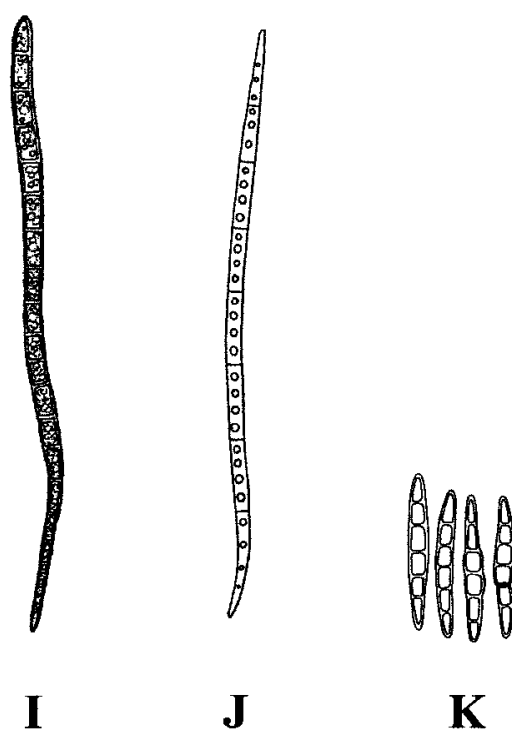

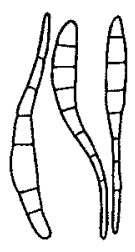

M

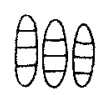

$\mathbf{N}$

FIG. 24. Diagrammatic representation of ascospores of Tubeufia species drawn to the same scale for comparison. A. T. acaciae (from Tilak and Kale 1970). B. T. aciculospora (from Katumoto and Harada 1979). C. T. aurantiella (from Rossman 1987). D. T. brevispina (from Barr and Rogerson 1983). E. T. cerea (from Barr 1980). F. T. claspisphaeria (this paper). G. T. cylindrothecia (from Barr 1980). H. T. dactylariae (from Chang 2003). I. T. eriodermae (from Etayo 2002). J. T. helicomyces (from Ellis and Ellis 1985). K. T. palmarum (from Samuels et al 1979). L. T. paludosa (from Sivanesan 1984). M. T. pannariae (from Etayo 2002). N. T. parvula (from Ellis and Ellis 1985). Bar $=20 \mu \mathrm{m}$. 
a glabrous surface or only short setae (FIG. 24). In ascospore size and morphology Tubeufia claspisphaeria is most similar to $T$. acaciae, T. pachythrix and $T$. stromaticola. It differs from these species in that it has a row of hook-like setae that form radially around the ostiole. The ascospores of $T$. claspisphaeria, T. pachythrix and T. stromaticola are elongate-fusiform, while those of T. acaciae are cylindrical or vermiform. Ascospores of T. claspisphaeria are wider than those of the other three species. Our collection of Tubeufia paludosa is similar to the description given by Barr (1980) but differs in that it has globose ascomata and mucilaginous pads at both ends of the ascospore.

Tubeufia species have rarely been reported from freshwater habitats (Shearer 1993) and are more commonly found on rotting vegetation (Barr 1980). Tubeufia paludosa previously has been reported from submerged grasses (Shearer 1993), T. cylindrothecia and T. palmarum from submerged wood (Ho et al 2001, Sivichai et al 2002). The anamorphs, however, commonly are reported from submerged freshwater litter (Ho et al 2002, Sivichai et al 2002).

\section{DICHOTOMOUS KEY TO THE SPECIES OF TUBEUFIA}

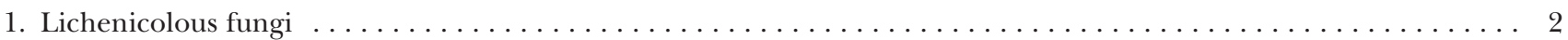

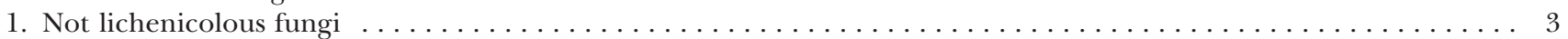
2. Ascomata white, ascospores $105-145 \times 4-5 \mu \mathrm{m}$, acicular, $17-19-$ septate $\ldots \ldots \ldots \ldots$. . . . . . . eriodermae

2. Ascomata orange-yellow to grayish-yellow, ascospores 40-50 $\times(3.5-4.5 \mu \mathrm{m}$, fusiform, 5-8-septate . . . T. pannariae

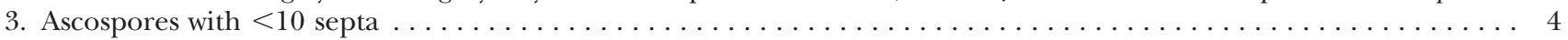

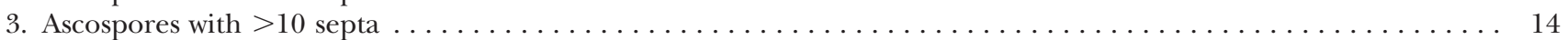

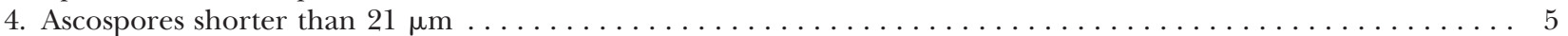

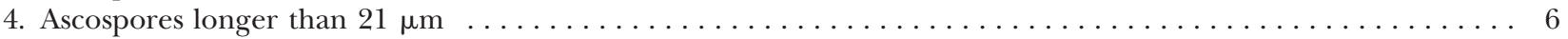

5. Ascomata reddish-brown, ascospores 14-15 $\times 3.5-4 \mu \mathrm{m}$, oblong-elliptical, 3-septate . . . . . . . . . . T. parvula

5. Ascomata hyaline to pale yellow, ascospores (13-) 14-18(-21) $\times(3.5-) 4-5(-7) \mu \mathrm{m}$, fusoid, 3-septate ... T. brevispina

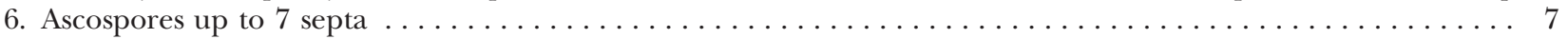

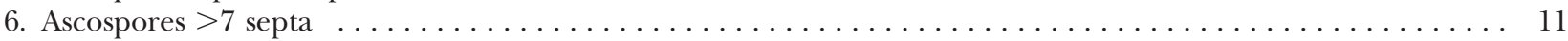

7. Ascospore $48-56 \times 6-7 \mu \mathrm{m}$, cylindrical or worm-like, often curved, pointed of both ends, 5-7-septate .... T. acaciae

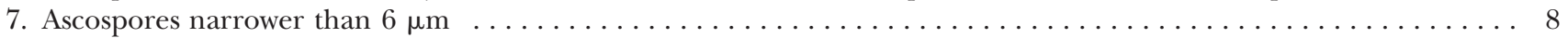

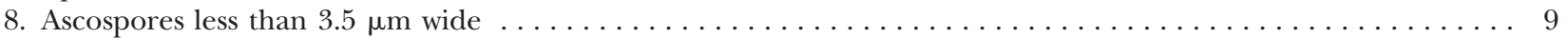

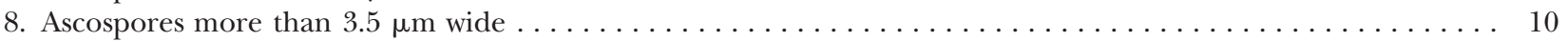

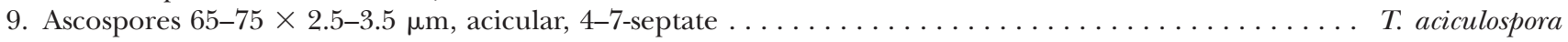

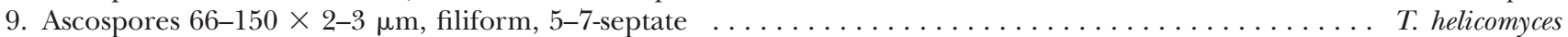
10. Ascospores 40-57 $\times 4-5 \mu \mathrm{m}$, long-fusiform, multiseptate (4-celled according to Rehm 1907) . . . . T. pachythrix 10. Ascospores $50-70 \times 4-5 \mu \mathrm{m}$, long-fusoid, $5-7$-septate $\ldots \ldots \ldots \ldots \ldots \ldots \ldots \ldots \ldots$ stromaticola

11. Ascomata with a row of hook-like setae, form radially around the ostiole, ascospores (34-) $45-60(-64) \times 5-7(-8) \mu \mathrm{m}$, elongate-fusiform, $(3-) 5-6(-7)$-septate $\ldots \ldots \ldots \ldots \ldots \ldots \ldots \ldots \ldots \ldots \ldots \ldots \ldots \ldots \ldots \ldots \ldots \ldots$

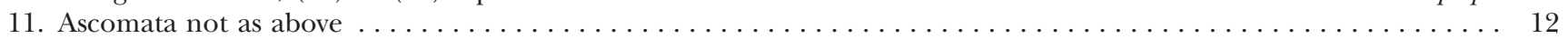

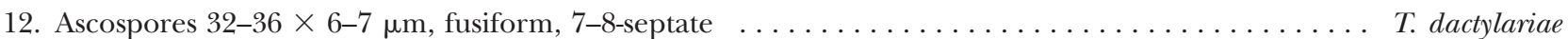

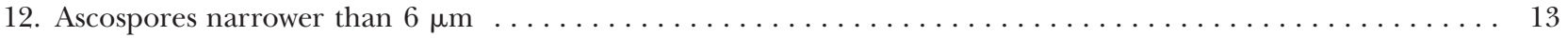

13. Ascospores $30-40(-50) \times 3-4 \mu \mathrm{m}$, fusiform with subacute to round ends, $5-7(-9)$-septate $\ldots \ldots$. . . T. palmarum

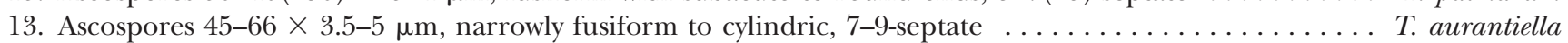

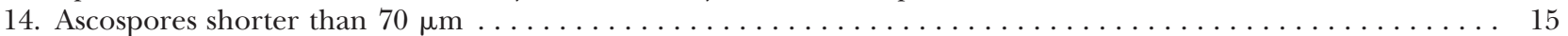
14. Ascospores (70-) 100-200(-230) $\times(2-) 3.5-7(-8) \mu \mathrm{m}$, fusiform with acute ends, up to 35 septa . . . . T. paludosa 15. Ascospores 40-55(-65) $\times(2.5-) 3-5 \mu \mathrm{m}$, elongate clavate or fusoid, (5-)7-9(-13)-septate . . . . . . T. cylindrothecia 15. Ascospores (27-) 30-52 × 2.5-3.5(-4.5) $\mu \mathrm{m}$, elongate fusoid, often curved, (5-)7-10(-13)-septate ........ T. cerea

\section{ACKNOWLEDGMENTS}

The senior author would like to thank Department of Ecology \& Biodiversity, The University of Hong Kong, for facilitating the work at the Centre for Research in Fungal Diversity. Thanks to Chiang Mai University Graduate School for partial support to the first author's doctoral study. Thanks also are extended to B. Bussaban, H.Y.M. Leung, M.H.W. Ng, I. Promputtha and S. Thongkantha for various help.

\section{LITERATURE CITED}

Barr ME. 1977. Acanthostigmella (Herpotrichiellaceae). Mycotaxon 6:17-23.

1980. On the family Tubeufiaceae (Pleosporales). Mycotaxon 12:137-167.

. 1987. Prodomus to class Loculoascomycetes. Amherst, Massachusetts: Published by the author. p 80-82.

, Rogerson CT. 1983. Two new species of Loculoascomycetes. Mycotaxon 17:247-252. 
Booth CE. 1964. Studies of Pyrenomycetes: VII. Mycol Pap 94:13-16.

Cai L, Tsui KM, Zhang K, Hyde KD. 2002. Aquatic fungi from Lake Fuxian, Yunnan, China. Fung Divers 9:5770 .

Chang HS. 2003. Tubeufia dactylariae sp. nov. and Acanthostigma scopulum, a new record of Taiwan. Bot Bull Acad Sin 44:253-256.

Choi YW, Hyde KD, Ho WH. 1999. Single spore isolation of fungi. Fung Divers 3:29-38.

Crane JL, Shearer CA, Barr ME. 1998. A revision of Boerlagiomyces with notes and a key to the saprobic genera of Tubeufiaceae. Canad J Bot 76:602-612.

Dennis RWG. 1986. Fungi of the Hebrides. Royal Botanical Garden, Kew. 383 pp.

Ellis JB, Everhart BM. 1892. The North American Pyrenomycetes. Newfield, New Jersey, U.S.A.: Published by authors. Johnson reprint corporation. 793 pp.

Ellis MB, Ellis JP. 1985. Microfungi on Land Plants: An Identification Handbook. Sydney, Australia: Published by authors. Croom Helm Australia Pty Ltd. 818 pp.

Etayo J. 2002. Aportación al conocimiento de los hongos liquenícolas de Colombia. Bibliotheca Lichenologica 84. Berlin, Stuttgart: J. Cramer. p 1-154.

Hino I, Katumoto K. 1954. Illustrationes fungorum bambusicolorum II. Bull Fac Agric Yamaguti Univ 5:212234.

Ho WH, Hyde KD, Hodgkiss IJ, Yanna. 2001. Fungal communities on submerged wood from streams in Brunei, Hong Kong, and Malaysia. Mycol Res 105:1492-1501.

—_ Yanna, Hyde KD, Hodgkiss IJ. 2002. Seasonality and sequential occurrence of fungi on wood submerged in Tai Po Kau Forest Stream, Hong Kong. Fung Divers 10: 21-43.

Hyde KD. 1993. Tropical Australian freshwater fungi. VI. Tiarosporella paludosa and Clohesyomyces aquaticus gen. et sp. nov. (Coelomycetes). Aust Syst Bot 6:169-173.

— - Yanna, Pinnoi A, Jones EBG. 2002. Goldanichiella fusiforma sp. nov. from palm fronds in Brunei and Thailand. Fung Divers 11:119-122.

Kirk PM, Cannon PF, David JC, Stalpers JA. 2001. Ainsworth and Bisby's Dictionary of the Fungi. 9th Edition. CABI Publishing.

Katumoto K, Harada Y. 1979. Plant parasitic fungi from the
Bonin Islands, Japan: 2. Ascomycotina and Deuteromycotina. Trans Mycol Soc Japan 20:411-428.

McKenzie EHC, Pinnoi A, Wong MKM, Hyde KD, Jones EBG. 2002. Two new hyaline Chalara species, and a key to species described since 1975. Fung Divers 11:129139.

Penzig O, Saccardo PA. 1897. Diagnoses fungorum novorum in insula Java collectorum. Malpighia 11:387-409.

Pirozynski KA. 1972. Microfungi of Tanzania. Mycol Pap 129:1-64.

Réblová M, Barr ME. 2000. The genus Acanthostigma (Tubeufiaceae, Pleosporales). Sydowia 52:258-285.

Rehm H. 1907. Ascomycetes novi. Annales Mycologici 5: 516-546.

Rossman AY. 1977. The genus Ophionectria (Euascomycetes, Hypocreales). Mycologia 69:355-391.

. 1979. A preliminary account of the taxa described in Calonectria. Mycotaxon 8:485-558.

- 1987. The Tubeufiaceae and similar Loculoascomycetes. Mycol Pap 157:1-71.

Samuels GJ, Müller E. 1978. Life history studies of Brazilian Ascomycetes 2. Sydowia 31:137-141.

— Brazilian Ascomycetes 6. Three species of Tubeufia with, respectively, dictyosporous-pycnidial and helicosporous anamorphs. Sydowia 31:180-192.

Scheuer C. 1991. Taphrophila (Dothideales: Tubeufiaceae) and two species of Tubeufia with dark setae. Mycol Res 95:811-816.

- 1993. Lectotypification of Ophiobolus trichellus (Dothideales, Ascomycetes). Mycotaxon 47:67-69.

Shearer CA. 1993. The freshwater Ascomycetes. Nova Hedwigia 56:1-33.

Sivanesan A. 1984. The bitunicate ascomycetes and their anamorphs. Vaduz, Germany: J. Cramer. 701 pp.

Sivichai S, Jones EBG, Hywel-Jones N. 2002. Fungal colonization of wood in a freshwater stream at Tad Ta Phu, Khao Yai National Park, Thailand. Fung Divers 10:113129.

Tilak ST, Kale SB. 1970. Contribution to our knowledge of ascomycetes of India-XIX. Sydowia 23:11-16.

Wong SW, Hyde KD. 1999. Proboscispora aquatica gen. et sp. nov. from wood submerged in freshwater. Mycol Res 103:81-87. 